\title{
Redundant Representation of Finite Fields
}

\author{
Willi Geiselmann and Harald Lukhaub
}

Institut für Algorithmen und Kognitive Systeme, Arbeitsgruppen Computeralgebra \& Systemsicherheit, Prof. Dr. Th. Beth, Universität Karlsruhe, Am Fasanengarten 5, 76128 Karlsruhe, Germany.

\begin{abstract}
A redundant representation of finite fields with $2^{n}$ elements is presented. It unifies the advantages of polynomial and normal bases by the cost of redundancy. The arithmetic, especially exponentiation, in this representation is perfectly suited for low power computing: multiplication can be built up with reversible gates very efficient and squaring is a cyclic shift.
\end{abstract}

\section{Introduction}

Hardware implementations of cryptographic schemes based on the Discrete Logarithm Problem in $\mathbb{F}_{2^{n}}$, the field with $2^{n}$ elements, (see e.g. 112), require efficient exponentiation architectures.

On the one hand, research has been particularly attracted to the choice of the representation of the field. Dual basis multipliers in $\mathbb{F}_{2^{n}}$ were first suggested by Berlekamp 14 for encoding Reed-Solomon codes. A representation in a polynomial basis is the standard representation, usually the best choice for generalpurpose applications. A very promising approach is the use of a normal basis, where squaring is an extremely simple operation 6 6 81113. However, normal basis multiplication can become very complex and good normal bases have to be selected carefully 13. Especially for exponentiation with low weight exponents the advantage of a normal basis is pronounced.

On the other hand, work on low power computing is coming up more and more. One reason is the problem of cooling of the chips with the increasing integration; another problem in cryptology is the resistance of encrption units against differential power analysis [9].

\subsection{Classical Multiplication}

In this section a brief overview on different representations of finite fields and the resulting circuits is given. More details can be found in [5,8]15].

Polynomial Basis Multiplication The standard representation of an extension field is the polynomial basis:

Definition 1. For $n \in \mathbb{N}, n \geq 2$ it holds $\mathbb{F}_{2^{n}} \simeq \mathbb{F}_{2}[x] / f(x)$ where $f(x) \in \mathbb{F}_{2}[x]$ is an irreducible polynomial of degree $n$. Let $\alpha \in \mathbb{F}_{2^{n}}$ be a zero of $f(x)$, then $\left(1, \alpha, \ldots, \alpha^{n-1}\right)$ is an $\mathbb{F}_{2}$ (vector space) basis of $\mathbf{F}_{2^{n}}$, called a polynomial basis. 
In this representation multiplication can easily be performed by linear feedback shift registers (LFSRs), where $f(x)$ is used as the feedback polynomial. One of the possible realizations is given in Figure 1.

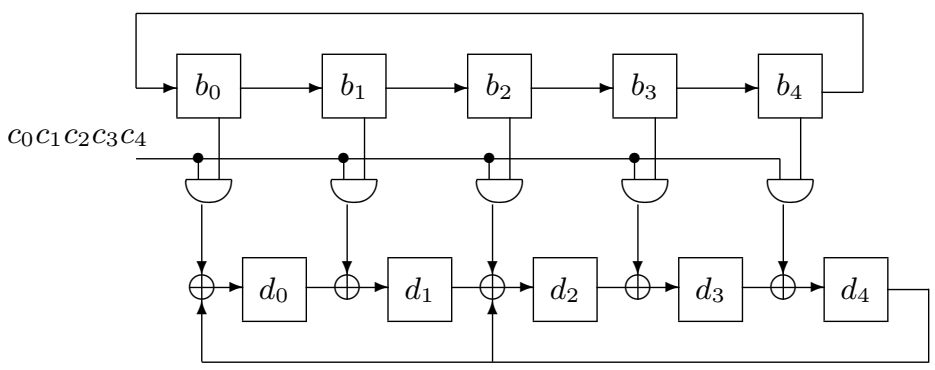

Fig. 1. A polynomial basis multiplier for $\mathbb{F}_{2^{5}}: \mathbb{F}_{2} \bmod f(x):=x^{5}+x^{2}+1$.

Normal Basis Multiplication A normal basis of $\mathbb{F}_{2^{n}}$ is defined as follows.

Definition 2. A basis $N=\left(\alpha_{0}, \alpha_{1}, \ldots, \alpha_{n-1}\right)$ of $\mathbb{F}_{2^{n}}$ is called a normal basis (over $\mathbb{F}_{2}$ ) if there exists some $\alpha \in \mathbb{F}_{2^{n}}$ with $\alpha_{i}=\alpha^{2^{i}}$ for all $i, 0 \leq i<n$.

In a normal basis $\left(\alpha_{0}, \alpha_{1}, \ldots, \alpha_{n-1}\right)$ of $\mathbb{F}_{2^{n}}$ squaring is a cyclic shift of coefficients due to

$$
\left(\sum_{i=0}^{n-1} u_{i} \alpha_{i}\right)^{2}=\sum_{i=0}^{n-1} u_{i} \alpha_{i+1} \quad \text { and } \alpha_{n}:=\alpha^{2^{n}}=\alpha_{0} .
$$

Multiplication is more difficult as the products $\alpha_{i} \alpha_{j}$ are, in general, not elements of the normal basis. The cost of normal basis multiplication is frequently measured by the complexity of the linear combinations needed to represent the elements $\alpha_{0} \alpha_{i}$ in the normal basis [13. The efficiency of any normal basis multiplier suggested uses field extensions in which good (optimal) normal bases exist [313]; in many fields such good normal bases are not available.

\subsection{Reversible Computing}

From physics it is known that erasure of information (deleting or resetting of storage cells) results in heating up the system. Therefore one approach to design low power computing devices is to use reversible gates [4,12,10]. These gates keep all information, i. e. they are bijections.

Permutations and shifts obviously fulfill this condition. 
One basic gate is the Fredkin gate: the inputs ( $a, b, c)$ are mapped to the outputs $(\mathrm{a}, \mathrm{b}, \mathrm{c})$ if $\mathrm{a}=0$; if the input $\mathrm{a}=1$ the outputs $\mathrm{b}, \mathrm{c}$ are exchanged, i. e. the output is ( $a, c, b)$.

Another reversible gate is the CNOT (Controlled NOT), the reversible version of the $\operatorname{XOR}((\mathrm{a}, \mathrm{b}) \mapsto(\mathrm{a}, \mathrm{a} \oplus \mathrm{b}))$. It is self inverse and can be built up with Fredkin gates.

The generalization of the CNOT is the Toffoli gate, it is a "double controlled $\operatorname{NOT}^{\prime \prime}((\mathrm{a}, \mathrm{b}, \mathrm{c}) \mapsto(\mathrm{a}, \mathrm{b}, \mathrm{c} \oplus(\mathrm{a} \wedge \mathrm{b})))$ and is self inverse.

All these gates are shown in Figure 2.

- Permutation \& Shift

- Fredkin

- CNOT

- Toffoli
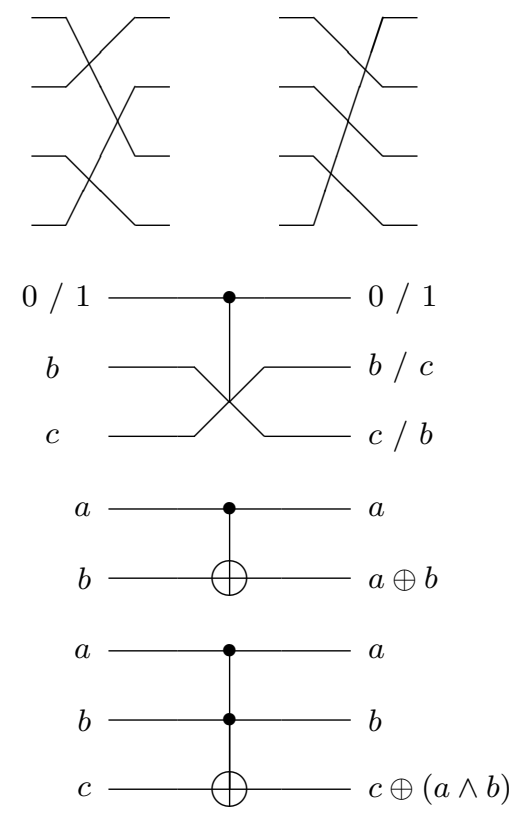

Fig. 2. Basic reversible gates

A polynomial basis multiplier, built up with reversible gates can be found in Figure 3. There are $n=5$ steps required, each step needs

$-2 \operatorname{shift}(n)$ operations;

$-\operatorname{wgt}(f(x))-2=1$ CNOT gates (the feedback operation) and

$-n=5$ Toffoli gates.

The normal basis multiplier suggested by Massey and Omura 11], modified for reversible gates, is shown in Figure 4. There are $n=5$ steps required, each step needs 


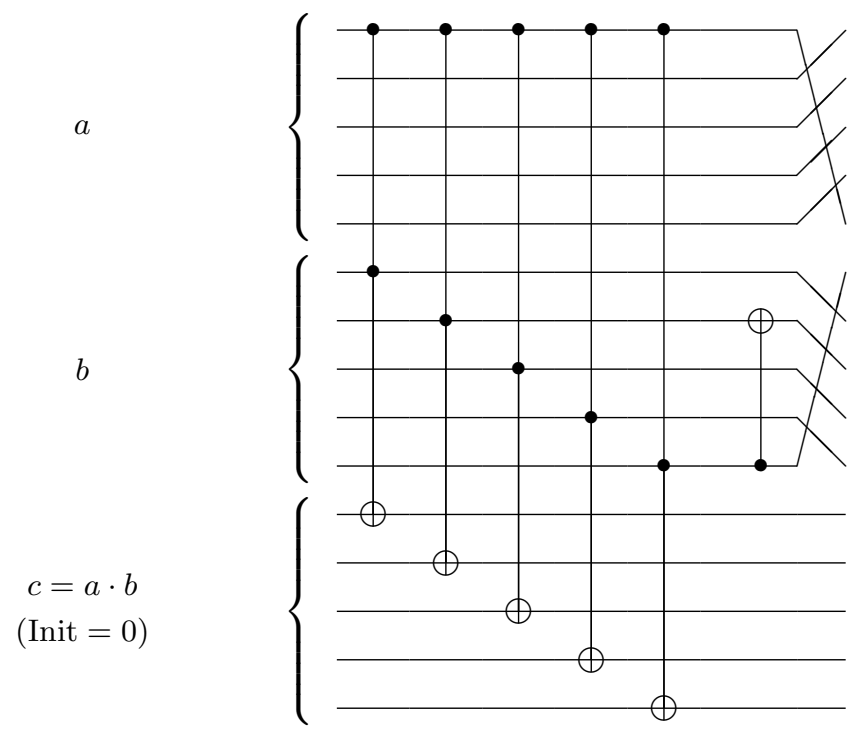

Fig. 3. A polynomial basis multiplier modulo $f(x)=x^{5}+x^{2}+1$

$-3 \operatorname{shift}(n)$ operations and

- "normal basis complexity $(f(x))$ " $=9$ Toffoli gates.

There are other realizations of normal basis multipliers, but the "normal basis complexity", as defined in [13, is a lower bound for the number of gates needed. This bound is for optimal normal bases of $\mathbb{F}_{2^{n}}$ equal to $2 n-1$. In most field extensions such a basis does not exist and in some cases the best known normal basis of $\mathbb{F}_{2^{n}}$ is not much smaller than $n^{2} / 2$. More details in this topic can be found in $6[8]$.

\section{Redundant Representation of Finite Fields}

The representation of $\mathbb{F}_{2^{n}}$ given in this section can perfectly be used in reversible circuits; but the representation can as well be used for very efficient classical circuits.

The main reason to use a normal basis is the easy procedure for squaring as a cyclic shift. The advantage of a polynomial basis is the easy realization of the multiplication as linear feedback shift register.

A representation that merges these two advantages is the following:

Theorem 1. Let $m \in \mathbf{N}$ be minimal with $x^{m}-1 \in \mathbb{F}_{2}[x]$ has an irreducible factor $f(x)$ of degree $n$; let $\alpha \in \mathbb{F}_{2^{n}}$ be a zero of $f(x)$. Then the elements of $\mathbb{F}_{2^{n}}$ can be represented as $\mathbb{F}_{2}$-vectors of size $m$ according to the following embedding: 


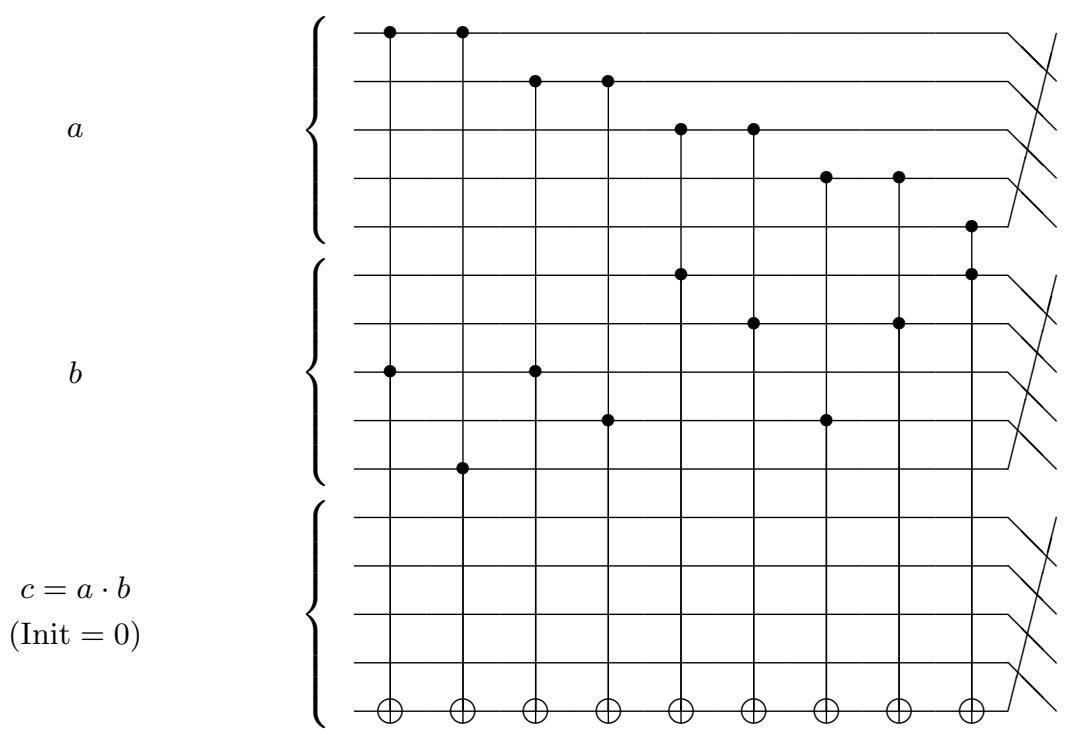

Fig. 4. The Massey-Omura normal basis multiplier with $f(x)=x^{5}+x^{4}+x^{2}+x+1$

$$
\begin{aligned}
& \mathbf{F}_{2}[x] / f(x) \underset{n-1}{\simeq} \mathbb{F}_{2}[\alpha] \hookrightarrow \mathbb{F}_{2}[x] /\left(x^{m}-1\right) \\
& \phi: \quad \sum_{i=0}^{n-1} a_{i} \cdot \alpha^{i} \mapsto \quad \sum_{i=0}^{n-1} a_{i} \cdot x^{i} \quad \hat{=}\left(a_{0}, \ldots, a_{n-1}, 0, \ldots, 0\right) \\
& \bar{\phi}: \quad \quad \quad \sum_{i=0}^{m-1} b_{i} \cdot \alpha^{i} \leftarrow \quad \sum_{i=0}^{m-1} b_{i} \cdot x^{i} \quad \hat{=} \quad\left(b_{0}, \ldots, b_{m-1}\right) .
\end{aligned}
$$

\section{It holds:}

(a) $\bar{\phi}(\phi(a)+\phi(b))=a+b \quad$ for $a, b \in \mathbb{F}_{2}[\alpha]$,

(b) $\bar{\phi}(\phi(a) \cdot \phi(b))=a \cdot b \quad$ for $a, b \in \mathbb{F}_{2}[\alpha]$.

Proof. Property (a) obviously holds.

To prove (b), note that $f(x) \mid\left(x^{m}-1\right)$ and thus $\alpha^{m}=1$.

The arithmetic in $\mathbb{F}_{2}[x] /\left(x^{n+1}+1\right)$, can be performed as follows:

- Addition: Is the XOR of the vectors.

- Multiplication with $x$ : Is a cyclic shift.

$$
\text { With } b:=\sum_{i=0}^{m-1} b_{i} \cdot x^{i} \text { it holds: } \begin{aligned}
b & \widehat{=}\left(b_{0}, b_{1}, \ldots, b_{m-1}\right) \\
\Rightarrow x \cdot b & \widehat{=}\left(b_{m-1}, b_{0}, \ldots, b_{m-2}\right) .
\end{aligned}
$$

- Multiplication: Is performed with an LFSR with trivial feedback polynomial $x^{m}-1$. 
- Squaring: Is a permutation ("stretching").

(Note $m$ is odd, because of $m$ being minimal with $f(x) \mid\left(x^{m}-1\right)$.) Let

$$
\begin{aligned}
b & :=\sum_{i=0}^{m-1} b_{i} \cdot x^{i}, \text { then } \\
b^{2} & =\sum_{i=0}^{m-1} b_{i} \cdot x^{2 i}=\sum_{i=0}^{(m-1) / 2} b_{i} \cdot x^{2 i}+\sum_{i=(m+1) / 2}^{m-1} b_{i} \cdot x^{2 i-m} .
\end{aligned}
$$

Written as vectors this is:

$$
\begin{aligned}
b & \widehat{=}\left(b_{0}, b_{1}, b_{2}, \ldots, b_{m-1}\right) \\
\Rightarrow b^{2} & \widehat{=}\left(b_{0}, b_{(m+1) / 2}, b_{1}, b_{(m+3) / 2}, b_{2}, \ldots, b_{m-1}, b_{(m-1) / 2}\right) .
\end{aligned}
$$

Example 1. The elements of the field $\mathbb{F}_{2^{4}}$ can be represented as vectors of length 5 according to the factorization of $x^{5}+1 \in \mathbb{F}_{2}[x]$ to $x^{5}+1=(x+1)\left(x^{4}+x^{3}+\right.$ $\left.x^{2}+x+1\right)$. Let $\alpha$ be a zero of $x^{4}+x^{3}+x^{2}+x+1$, then $\left(1+\alpha^{3}\right)^{3}$ can be calculated as follows:

$$
\begin{aligned}
\mathbb{F}_{2}[\alpha] & \hookrightarrow \mathbb{F}_{2}\left[x /\left(x^{5}+1\right)\right. \\
1+\alpha^{3} & \hat{=}(1,0,0,1,0) \\
\left(1+\alpha^{3}\right)^{2} & \hat{=}(1,1,0,0,0) \quad \text { (by stretching) } \\
\left(1+\alpha^{3}\right)^{2} \cdot\left(1+\alpha^{3}\right) & \hat{=}(1,1,0,0,0) \oplus(0,0,0,1,1) \\
& \hat{=}(1,1,0,1,1) \quad\left(\text { with } \alpha^{4}=\alpha^{3}+\alpha^{2}+\alpha+1\right. \\
\alpha^{2} & \hat{=}(1,1,0,1,1) .
\end{aligned}
$$

In the appendix a list of the minimal required length $m$ of the redundant representation is given for $n \leq 250$ and for primes $n \leq 2000$.

\section{Multiplication with a Fixed Element}

For crypto systems based on a discrete logarithm problem in $\mathbb{F}_{2^{n}}$ exponentiation of one fixed element $a \in \mathbb{F}_{2^{n}}$ is one of the operations required. This is an operation perfectly suited to reversible computing when squaring is a permutation. The multiplication with $a$ is a linear mapping and can be performed without any intermediate results that need to be deleted.

One way to generate a reversible implementation of a linear mapping (represented as multiplication $\phi(a) \mapsto A \cdot \phi(a)$ with $A$ an $m \times m$ matrix and $\phi(a)$ a vector of size $m$ ) is to decompose $A$ into $A=P \cdot L \cdot U$ with $P$ a permutation matrix, $L$ a lower triangular matrix and $U$ an upper triangular matrix.

For the multiplication $U \cdot \phi(a)$ first calculate the first component by adding the required components to it (first row of $U$ ). After this step one bit of the result is stored in the first component - the previous value is lost, but not needed any more because of the trinagular structere of $U$. Proceeding with the other components of $\phi(a)$ in the same way gives $U \cdot \phi(a)$ without any intermediate 
result to be deleted. The number of CNOT gates required is the weight of $U-I_{m}$, where $I_{m}$ denotes the unity matrix of size $m$.

The multiplication with $L$ is performed in the same way, but the resulting bits are calculated in the reverse order.

In the rest of this section we give some examples for normal basis multiplication and the multiplication in the corresponding redundant representation with reversible gates according to the decomposition of matrices.

Example 2. For the smaller examples the decomposition of the matrices is given, for the larger ones we restrict ourself to the complexity only.

$-\mathbb{F}_{2^{4}}$ :

Let $\alpha$ be a zero of $x^{4}+x^{3}+x^{2}+x+1$, then $\alpha$ generates a normal basis with the multiplication matrix

$$
A_{4}:=\left(\begin{array}{cccc}
0 & 1 & 0 & 0 \\
0 & 0 & 0 & 1 \\
1 & 1 & 1 & 1 \\
0 & 0 & 1 & 0
\end{array}\right)
$$

Then $\alpha+1$ is a primitive element and the multiplication with $A_{4}+I_{4}$ decomposes as follows:

$$
A_{4}+I_{4}=\left(\begin{array}{llll}
1 & 0 & 0 & 0 \\
0 & 1 & 0 & 0 \\
0 & 0 & 0 & 1 \\
0 & 0 & 1 & 0
\end{array}\right) \cdot\left(\begin{array}{llll}
1 & 0 & 0 & 0 \\
0 & 1 & 0 & 0 \\
0 & 0 & 1 & 0 \\
1 & 0 & 0 & 1
\end{array}\right) \cdot\left(\begin{array}{llll}
1 & 1 & 0 & 0 \\
0 & 1 & 0 & 1 \\
0 & 0 & 1 & 1 \\
0 & 0 & 0 & 1
\end{array}\right)
$$

This decomposition results in a multiplication circuit with $1+3=4$ CNOT gates and 1 permutation of two elements.

For the redundant representation $m=5$ (note: $x^{5}=(x+1)\left(x^{4}+x^{3}+\right.$ $\left.x^{2}+x+1\right)$ ). The matrix $R_{4} \in \mathbb{F}_{2}^{5 \times 5}$ corresponding to multiplication with $x$ has to perform a cyclic shift and thus is the circulant matrix with first row $(0,1,0,0,0)$. The element $1+x+x^{2}$ is primitive, and the decomposition of the corresponding matrix is:

$$
I_{5}+R_{4}+R_{4}^{2}=\left(\begin{array}{lllll}
1 & 0 & 0 & 0 & 0 \\
0 & 1 & 0 & 0 & 0 \\
0 & 0 & 1 & 0 & 0 \\
0 & 0 & 0 & 0 & 1 \\
0 & 0 & 0 & 1 & 0
\end{array}\right) \cdot\left(\begin{array}{lllll}
1 & 0 & 0 & 0 & 0 \\
0 & 1 & 0 & 0 & 0 \\
0 & 0 & 1 & 0 & 0 \\
1 & 0 & 1 & 1 & 0 \\
1 & 1 & 0 & 0 & 1
\end{array}\right) \cdot\left(\begin{array}{lllll}
1 & 1 & 1 & 0 & 0 \\
0 & 1 & 1 & 1 & 0 \\
0 & 0 & 1 & 1 & 1 \\
0 & 0 & 0 & 1 & 0 \\
0 & 0 & 0 & 0 & 1
\end{array}\right) .
$$

Here the multiplication circuit requires $4+6=10$ CNOT gates and 1 permutation of two elements. 
$-\mathbb{F}_{2^{6}}$ :

In this field an optimal normal basis exists with the multiplication matrix:

$$
A_{6}:=\left(\begin{array}{cccccc}
0 & 1 & 0 & 0 & 0 & 0 \\
1 & 0 & 0 & 0 & 1 & 0 \\
0 & 0 & 0 & 1 & 1 & 0 \\
0 & 0 & 1 & 0 & 0 & 1 \\
0 & 1 & 1 & 0 & 0 & 0 \\
0 & 0 & 0 & 1 & 0 & 1
\end{array}\right)
$$

The corresponding field element is primitive and $A_{6}$ decomposes to:

$$
A_{6}=\left(\begin{array}{llllll}
0 & 1 & 0 & 0 & 0 & 0 \\
1 & 0 & 0 & 0 & 0 & 0 \\
0 & 0 & 0 & 1 & 0 & 0 \\
0 & 0 & 1 & 0 & 0 & 0 \\
0 & 0 & 0 & 0 & 0 & 1 \\
0 & 0 & 0 & 0 & 1 & 0
\end{array}\right) \cdot\left(\begin{array}{llllll}
1 & 0 & 0 & 0 & 0 & 0 \\
0 & 1 & 0 & 0 & 0 & 0 \\
0 & 0 & 1 & 0 & 0 & 0 \\
0 & 0 & 0 & 1 & 0 & 0 \\
0 & 0 & 0 & 1 & 1 & 0 \\
0 & 1 & 1 & 0 & 0 & 1
\end{array}\right) \cdot\left(\begin{array}{llllll}
1 & 0 & 0 & 0 & 1 & 0 \\
0 & 1 & 0 & 0 & 0 & 0 \\
0 & 0 & 1 & 0 & 0 & 1 \\
0 & 0 & 0 & 1 & 1 & 0 \\
0 & 0 & 0 & 0 & 1 & 1 \\
0 & 0 & 0 & 0 & 0 & 1
\end{array}\right),
$$

giving a reversible realization with $3+4=7$ CNOT gates and 3 permutations of two elements.

For the redundant representation $m=9$ (note: $x^{9}+1=(x+1)\left(x^{2}+x+\right.$ $\left.1)\left(x^{6}+x^{3}+1\right)\right)$. The element $1+x+x^{3}$ is primitive, and the decomposition of the corresponding matrix is:

$$
I_{9}+R_{6}+R_{6}^{3}=\left(\begin{array}{lllllllll}
1 & 0 & 0 & 0 & 0 & 0 & 0 & 0 & 0 \\
0 & 1 & 0 & 0 & 0 & 0 & 0 & 0 & 0 \\
0 & 0 & 1 & 0 & 0 & 0 & 0 & 0 & 0 \\
0 & 0 & 0 & 1 & 0 & 0 & 0 & 0 & 0 \\
0 & 0 & 0 & 0 & 1 & 0 & 0 & 0 & 0 \\
0 & 0 & 0 & 0 & 0 & 1 & 0 & 0 & 0 \\
0 & 0 & 0 & 0 & 0 & 0 & 1 & 0 & 0 \\
0 & 0 & 0 & 0 & 0 & 0 & 0 & 1 & 0 \\
0 & 0 & 0 & 0 & 0 & 0 & 0 & 0 & 1
\end{array}\right) \cdot\left(\begin{array}{lllllllll}
1 & 0 & 0 & 0 & 0 & 0 & 0 & 0 & 0 \\
0 & 1 & 0 & 0 & 0 & 0 & 0 & 0 & 0 \\
0 & 0 & 1 & 0 & 0 & 0 & 0 & 0 & 0 \\
0 & 0 & 0 & 1 & 0 & 0 & 0 & 0 & 0 \\
0 & 0 & 0 & 0 & 1 & 0 & 0 & 0 & 0 \\
0 & 0 & 0 & 0 & 0 & 1 & 0 & 0 & 0 \\
1 & 1 & 1 & 0 & 1 & 0 & 1 & 0 & 0 \\
0 & 1 & 1 & 1 & 0 & 1 & 0 & 1 & 0 \\
1 & 1 & 0 & 1 & 0 & 0 & 1 & 0 & 1
\end{array}\right) \cdot\left(\begin{array}{lllllllll}
1 & 1 & 0 & 1 & 0 & 0 & 0 & 0 & 0 \\
0 & 1 & 1 & 0 & 1 & 0 & 0 & 0 & 0 \\
0 & 0 & 1 & 1 & 0 & 1 & 0 & 0 & 0 \\
0 & 0 & 0 & 1 & 1 & 0 & 1 & 0 & 0 \\
0 & 0 & 0 & 0 & 1 & 1 & 0 & 1 & 0 \\
0 & 0 & 0 & 0 & 0 & 1 & 1 & 0 & 1 \\
0 & 0 & 0 & 0 & 0 & 0 & 1 & 0 & 0 \\
0 & 0 & 0 & 0 & 0 & 0 & 0 & 1 & 0 \\
0 & 0 & 0 & 0 & 0 & 0 & 0 & 0 & 1
\end{array}\right) .
$$

Here the multiplication circuit requires $12+12=24$ CNOT gates and no permutation.

$-\mathbb{F}_{2^{54}}$ :

In $\mathbb{F}_{2^{54}}$ a good normal basis (with complexity 209) exists. The generator $\alpha$ is not primitive, thus we choose the primitive Element: $1+\alpha$. The decomposition of the corresponding matrix gives us a circuit with $236+255=491$ CNOT gates and a very irregular permutation.

The redundant representation with $m=81$ and the primitive element $x+$ $x^{3}+x^{4}$ gives a circuit with $135+159=294$ CNOT gates and a very regular permutation, a cyclic shift on all but 3 elements. 
$-\mathbb{F}_{2^{121}}$ :

In $\mathbb{F}_{2^{121}}$ a good normal basis (with complexity 705) exists. The generator $\alpha$ is primitive and the decomposition of the corresponding matrix gives us a circuit with $1183+1126=2309$ CNOT gates and a very irregular permutation.

The redundant representation with $m=727$ and the primitive element $x+$ $x^{2}+x^{3}$ gives a circuit with $968+550=1518$ CNOT gates and a very regular permutation, close to a cyclic shift.

$-\mathbb{F}_{2^{239}}$ :

In $\mathbb{F}_{2^{239}}$ a good normal basis (with complexity 477 ) exists. The generator $\alpha$ is primitive and the decomposition of the corresponding matrix gives us a circuit with $233+234=467$ CNOT gates and a very irregular permutation.

The redundant representation with $m=479$ and the primitive element $x+$ $x^{2}+x^{3}$ gives a circuit with $637+955=1592$ CNOT gates and a very regular permutation, close to a cyclic shift.

$-\mathbb{F}_{2^{240}}$ :

In $\mathbb{F}_{2^{240}}$ no good normal basis exists. We chose the best we found, with complexity 28433. The generator $\alpha$ is primitive and the decomposition of the corresponding matrix gives us a circuit with $14213+14370=28583$ CNOT gates and a very irregular permutation.

The redundant representation with $m=1067$ and the primitive element $1+x+x^{2}$ gives a circuit with $1420+2130=3550$ CNOT gates and a very regular permutation, close to a cyclic shift.

If a good normal bases exists in a field extension, it depends very much on the degree of redundancy required, if the normal basis representation or the redundant representation is better. If an optimal normal basis of type 1 (see. [13]) exists for the extension degree $n$, the redundant representation with $m=n+1$ is the best choice for implementation; if an optimal normal basis of type 2 (see. [13]) exists, the best redundant representation is with $m=2 n-1$ and the normal basis multiplication (with complexity $2 n-1$ ) is the better choice.

If no good normal basis exists in the field in question even a high degree of redundancy will result in a better performance than a normal basis multiplier.

\section{General Multiplication}

The general multiplication (with two variable inputs) can be realized with reversible gates in a straight forward manner. There are exactly $m$ Toffoli gates and 2 cyclic shifts of $m$ signals needed. It corresponds directly to a LFSR with trivial feedback polynomial. 
The major problem of any normal basis multiplier is the irregular structure. In hardware it results in additional area needed for wireing, in software unregular access to the bits causes additional operations. With the redundant representation these problems do not occure at all. The highly regular structure can be seen best in some example: The field $\mathbb{F}_{2^{4}}$ is represented as $\mathbb{F}_{2}[x] /\left(x^{5}-x\right)$ :

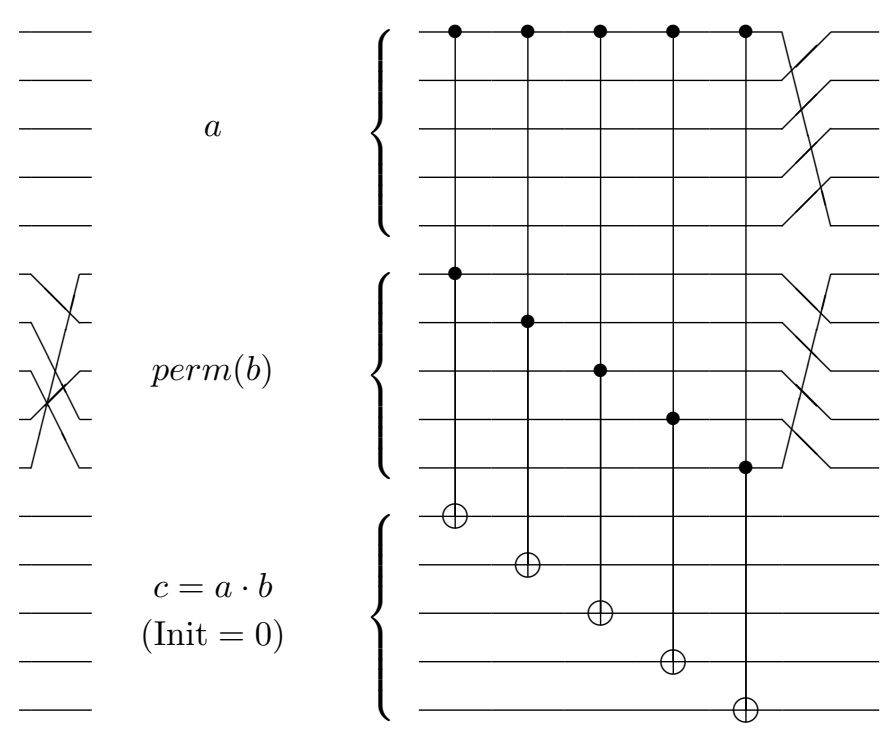

The corresponding normal basis multiplier needs "normal basis complexity $(f(x))$ " many Toffoli gates, in this example this is 9 . The permutation of the input $b$ seems to reduce the advantage of the redundant representation; in cryptography in most cases an exponentiation is required, and thus the permutation has to be performed only once and not for each multiplication.

In this setting the redundant representation has often a better performance than a normal basis representation if the number of gates is the measure to be used. If the regularity of the design is taken into account the advantage is even more pronounced.

\section{Conclusion}

The presented redundant representation of finite extension fields via roots of unity joins the two good properties of normal and polynomial bases. On the one hand squaring can be performed by a simple and very structured permutation a stretching; on the other hand multiplication can be performed with an LFSR with trivial feedback polynomial.

The price to pay is the additional place required for storage of the redundancy. The level of redundancy depends very much on the degree of the field and in many cases it is a perfect choice for exponentiation. 


\section{A Degree of Redundancy}

In the following table for all fields $\mathbb{F}_{2^{n}}$ with $n \leq 250$ and extension degrees with $n \leq 2000$ beeing a prime, the smallest degree of redundancy $m$ is given, that allows a representation with roots of unity. In some cases even the field $\mathbb{F}_{2^{n_{1}}} \supset \mathbb{F}_{2^{n}}$ can be represented with the smallest $m$-th roots of unity where $\mathbb{F}_{2^{n}}$ can be embedded.

\begin{tabular}{|c|c|c|c|c|c|}
\hline$n$ & $n_{1}$ & $m$ & $n$ & $n_{1}$ & $m$ \\
\hline & & & 41 & 82 & 83 \\
\hline 2 & 2 & 3 & 42 & 42 & 147 \\
\hline 3 & 3 & 7 & 43 & 172 & 173 \\
\hline 4 & 4 & 5 & 44 & 44 & 115 \\
\hline 5 & 10 & 11 & 45 & 180 & 181 \\
\hline 6 & 6 & 9 & 46 & 138 & 139 \\
\hline 7 & 28 & 29 & 47 & 94 & 283 \\
\hline 8 & 8 & 17 & 48 & 48 & 97 \\
\hline 9 & 18 & 19 & 49 & 196 & 197 \\
\hline 10 & 10 & 11 & 50 & 100 & 101 \\
\hline 11 & 11 & 23 & 51 & 51 & 103 \\
\hline 12 & 12 & 13 & 52 & 52 & 53 \\
\hline 13 & 52 & 53 & 53 & 106 & 107 \\
\hline 14 & 28 & 29 & 54 & 54 & 81 \\
\hline 15 & 60 & 61 & 55 & 110 & 121 \\
\hline 16 & 48 & 97 & 56 & 224 & 449 \\
\hline 17 & 51 & 103 & 57 & 342 & 361 \\
\hline 18 & 18 & 19 & 58 & 58 & 59 \\
\hline 19 & 95 & 191 & 59 & 708 & 709 \\
\hline 20 & 20 & 25 & 60 & 60 & 61 \\
\hline 21 & 21 & 49 & 61 & 183 & 367 \\
\hline 22 & 66 & 67 & 62 & 372 & 373 \\
\hline 23 & 23 & 47 & 63 & 378 & 379 \\
\hline 24 & 48 & 97 & 64 & 64 & 641 \\
\hline 25 & 100 & 101 & 65 & 130 & 131 \\
\hline 26 & 52 & 53 & 66 & 66 & 67 \\
\hline 27 & 54 & 81 & 67 & 268 & 269 \\
\hline 28 & 28 & 29 & 68 & 68 & 137 \\
\hline 29 & 58 & 59 & 69 & 138 & 139 \\
\hline 30 & 60 & 61 & 70 & 210 & 211 \\
\hline 31 & 155 & 311 & 71 & 284 & 569 \\
\hline 32 & 96 & 193 & 72 & 72 & 323 \\
\hline 33 & 66 & 67 & 73 & 292 & 293 \\
\hline 34 & 68 & 137 & 74 & 148 & 149 \\
\hline 35 & 35 & 71 & 75 & 300 & 707 \\
\hline 36 & 36 & 37 & 76 & 76 & 229 \\
\hline 37 & 148 & 149 & 77 & 231 & 463 \\
\hline 38 & 76 & 229 & 78 & 156 & 169 \\
\hline 39 & 39 & 79 & 79 & 316 & 317 \\
\hline 40 & 40 & 187 & 80 & 240 & 1067 \\
\hline
\end{tabular}

\begin{tabular}{r|r|r}
$r$ & $n_{1}$ & \multicolumn{1}{|c}{$m$} \\
\hline 81 & 162 & 163 \\
82 & 82 & 83 \\
83 & 83 & 167 \\
84 & 84 & 203 \\
85 & 340 & 1021 \\
86 & 172 & 173 \\
87 & 348 & 349 \\
88 & 88 & 353 \\
89 & 178 & 179 \\
90 & 180 & 181 \\
91 & 546 & 547 \\
92 & 92 & 235 \\
93 & 372 & 373 \\
94 & 94 & 283 \\
95 & 95 & 191 \\
96 & 96 & 193 \\
97 & 388 & 389 \\
98 & 196 & 197 \\
99 & 99 & 199 \\
100 & 100 & 101 \\
101 & 303 & 607 \\
102 & 102 & 307 \\
103 & 618 & 619 \\
104 & 104 & 901 \\
105 & 210 & 211 \\
106 & 106 & 107 \\
107 & 214 & 643 \\
108 & 108 & 405 \\
109 & 1090 & 1091 \\
110 & 110 & 121 \\
111 & 444 & 1043 \\
112 & 224 & 449 \\
113 & 226 & 227 \\
114 & 342 & 361 \\
115 & 460 & 461 \\
116 & 116 & 295 \\
117 & 117 & 937 \\
118 & 708 & 709 \\
119 & 119 & 239 \\
120 & 120 & 1037
\end{tabular}




\begin{tabular}{|c|c|c|c|c|c|}
\hline$n$ & $n_{1}$ & $m$ & $n$ & $n_{1}$ & $m$ \\
\hline 161 & 483 & 967 & 206 & 618 & 619 \\
\hline 162 & 162 & 163 & 207 & 828 & 829 \\
\hline 163 & 652 & 653 & 208 & 1040 & 2081 \\
\hline 164 & 164 & 415 & 209 & 418 & 419 \\
\hline 165 & 660 & 661 & 210 & 210 & 211 \\
\hline 166 & 166 & 499 & 211 & 1055 & 2111 \\
\hline 167 & 2338 & 2339 & 212 & 212 & 535 \\
\hline 168 & 168 & 833 & 213 & 852 & 853 \\
\hline 169 & 676 & 677 & 214 & 214 & 643 \\
\hline 170 & 340 & 1021 & 215 & 1290 & 1291 \\
\hline 171 & 342 & 361 & 216 & 648 & 1297 \\
\hline 172 & 172 & 173 & 217 & 651 & 1303 \\
\hline 173 & 346 & 347 & 218 & 1090 & 1091 \\
\hline 174 & 348 & 349 & 219 & 876 & 877 \\
\hline 175 & 700 & 701 & 220 & 220 & 575 \\
\hline 176 & 704 & 1409 & 221 & 442 & 443 \\
\hline 177 & 708 & 709 & 222 & 444 & 1043 \\
\hline 178 & 178 & 179 & 223 & 2676 & 2677 \\
\hline 179 & 179 & 359 & 224 & 224 & 449 \\
\hline 180 & 180 & 181 & 225 & 900 & 1919 \\
\hline 181 & 543 & 1087 & 226 & 226 & 227 \\
\hline 182 & 546 & 547 & 227 & 908 & 5449 \\
\hline 183 & 183 & 367 & 228 & 228 & 1603 \\
\hline 184 & 184 & 799 & 229 & 916 & 2749 \\
\hline 185 & 370 & 1481 & 230 & 460 & 461 \\
\hline 186 & 372 & 373 & 231 & 231 & 463 \\
\hline 187 & 1122 & 1123 & 232 & 464 & 929 \\
\hline 188 & 940 & 941 & 233 & 466 & 467 \\
\hline 189 & 378 & 379 & 234 & 468 & 1007 \\
\hline 190 & 190 & 573 & 235 & 940 & 941 \\
\hline 191 & 191 & 383 & 236 & 708 & 709 \\
\hline 192 & 384 & 769 & 237 & 237 & 1423 \\
\hline 193 & 772 & 773 & 238 & 238 & 717 \\
\hline 194 & 388 & 389 & 239 & 239 & 479 \\
\hline 195 & 390 & 869 & 240 & 240 & 1067 \\
\hline 196 & 196 & 197 & 241 & 723 & 1447 \\
\hline 197 & 3546 & 3547 & 242 & 1210 & 1331 \\
\hline 198 & 198 & 437 & 243 & 243 & 487 \\
\hline 199 & 796 & 797 & 244 & 244 & 733 \\
\hline 200 & 200 & 401 & 245 & 490 & 491 \\
\hline 201 & 201 & 1609 & 246 & 246 & 581 \\
\hline 202 & 404 & 809 & 247 & 1482 & 1483 \\
\hline 203 & 812 & 841 & 248 & 744 & 1489 \\
\hline 204 & 204 & 409 & 249 & 249 & 1169 \\
\hline 205 & 820 & 821 & 250 & 500 & 625 \\
\hline
\end{tabular}

\begin{tabular}{|c|c|c|c|c|c|}
\hline$n$ & $n_{1}$ & $m$ & $n$ & $n_{1}$ & $m$ \\
\hline 251 & 251 & 503 & 523 & 2615 & 5231 \\
\hline 257 & 771 & 1543 & 541 & 3246 & 9739 \\
\hline 263 & 526 & 1579 & 547 & 547 & 5471 \\
\hline 269 & 1076 & 2153 & 557 & 557 & 3343 \\
\hline 271 & 542 & 1627 & 563 & 7882 & 7883 \\
\hline 277 & 1108 & 1109 & 569 & 6828 & 6829 \\
\hline 281 & 562 & 563 & 571 & 571 & 5711 \\
\hline 283 & 566 & 1699 & 577 & 2308 & 2309 \\
\hline 293 & 586 & 587 & 587 & 8218 & 8219 \\
\hline 307 & 1228 & 1229 & 593 & 1186 & 1187 \\
\hline 311 & 1866 & 1867 & 599 & 2396 & 4793 \\
\hline 313 & 939 & 1879 & 601 & 601 & 3607 \\
\hline 317 & 8242 & 8243 & 607 & 3642 & 3643 \\
\hline 331 & 1986 & 1987 & 613 & 6130 & 6131 \\
\hline 337 & 3370 & 3371 & 617 & 1234 & 4937 \\
\hline 347 & 2082 & 2083 & 619 & 2476 & 2477 \\
\hline 349 & 3490 & 3491 & 631 & 3155 & 6311 \\
\hline 353 & 2471 & 4943 & 641 & 1282 & 1283 \\
\hline 359 & 359 & 719 & 643 & 7716 & 7717 \\
\hline 367 & 734 & 2203 & 647 & 9058 & 9059 \\
\hline 373 & 1492 & 1493 & 653 & 1306 & 1307 \\
\hline 379 & 1516 & 4549 & 659 & 659 & 1319 \\
\hline 383 & 1532 & 4597 & 661 & 1983 & 3967 \\
\hline 389 & 2334 & 9337 & 673 & 2692 & 2693 \\
\hline 397 & 397 & 2383 & 677 & 2708 & 5417 \\
\hline 401 & 1604 & 3209 & 683 & 683 & 1367 \\
\hline 409 & 1636 & 1637 & 691 & 3455 & 6911 \\
\hline 419 & 419 & 839 & 701 & 12618 & 12619 \\
\hline 421 & 842 & 4211 & 709 & 2836 & 2837 \\
\hline 431 & 431 & 863 & 719 & 719 & 1439 \\
\hline 433 & 1732 & 1733 & 727 & 2908 & 2909 \\
\hline 439 & 2195 & 4391 & 733 & 7330 & 7331 \\
\hline 443 & 443 & 887 & 739 & 2956 & 2957 \\
\hline 449 & 1796 & 3593 & 743 & 743 & 1487 \\
\hline 457 & 6855 & 13711 & 751 & 4506 & 4507 \\
\hline 461 & 461 & 2767 & 757 & 6056 & 12113 \\
\hline 463 & 5556 & 5557 & 761 & 1522 & 1523 \\
\hline 467 & 2802 & 2803 & 769 & 7690 & 7691 \\
\hline 479 & 958 & 3833 & 773 & 2319 & 4639 \\
\hline 487 & 1948 & 1949 & 787 & 4722 & 4723 \\
\hline 491 & 491 & 983 & 797 & 2391 & 4783 \\
\hline 499 & 1996 & 1997 & 809 & 1618 & 1619 \\
\hline 503 & 3018 & 3019 & 811 & 4055 & 8111 \\
\hline 509 & 1018 & 1019 & 821 & 1642 & 6569 \\
\hline 521 & 2084 & 16673 & 823 & 4115 & 8231 \\
\hline
\end{tabular}




\begin{tabular}{r|r|r}
\multicolumn{1}{r|}{$n$} & \multicolumn{1}{|r}{$n_{1}$} & \multicolumn{1}{|c}{$m$} \\
\hline 827 & 11578 & 11579 \\
829 & 8290 & 8291 \\
839 & 10068 & 10069 \\
853 & 3412 & 3413 \\
857 & 857 & 6857 \\
859 & 18898 & 18899 \\
863 & 5178 & 5179 \\
877 & 7016 & 14033 \\
881 & 15858 & 15859 \\
883 & 3532 & 3533 \\
887 & 1774 & 5323 \\
907 & 5442 & 5443 \\
911 & 911 & 1823 \\
919 & 3676 & 3677 \\
929 & 3716 & 7433 \\
937 & 2811 & 5623 \\
941 & 2823 & 5647 \\
947 & 5682 & 5683 \\
953 & 1906 & 1907 \\
967 & 7736 & 15473 \\
971 & 5826 & 5827 \\
977 & 1954 & 7817 \\
983 & 13762 & 13763 \\
991 & 8919 & 17839 \\
997 & 3988 & 3989 \\
1009 & 10090 & 10091 \\
1013 & 2026 & 2027 \\
1019 & 1019 & 2039 \\
1021 & 2042 & 10211 \\
1031 & 1031 & 2063 \\
1033 & 4132 & 4133 \\
1039 & 4156 & 4157 \\
1049 & 2098 & 2099 \\
1051 & 12612 & 12613 \\
1061 & 3183 & 6367 \\
1063 & 4252 & 4253 \\
1069 & 10690 & 10691 \\
1087 & 4348 & 4349 \\
1091 & 6546 & 6547 \\
1093 & 4372 & 4373
\end{tabular}

\begin{tabular}{r|r|r}
\multicolumn{1}{r|}{$n$} & \multicolumn{1}{|c|}{$n_{1}$} & \multicolumn{1}{|c}{$m$} \\
\hline 1097 & 7679 & 15359 \\
1103 & 1103 & 2207 \\
1109 & 4436 & 13309 \\
1117 & 3351 & 6703 \\
1123 & 4492 & 4493 \\
1129 & 4516 & 4517 \\
1151 & 6906 & 6907 \\
1153 & 12683 & 25367 \\
1163 & 4652 & 37217 \\
1171 & 7026 & 7027 \\
1181 & 14172 & 14173 \\
1187 & 4748 & 9497 \\
1193 & 3579 & 7159 \\
1201 & 3603 & 7207 \\
1213 & 14556 & 14557 \\
1217 & 3651 & 29209 \\
1223 & 1223 & 2447 \\
1229 & 2458 & 2459 \\
1231 & 9848 & 19697 \\
1237 & 2474 & 19793 \\
1249 & 12490 & 12491 \\
1259 & 17626 & 17627 \\
1277 & 25540 & 25541 \\
1279 & 6395 & 12791 \\
1283 & 2566 & 7699 \\
1289 & 2578 & 2579 \\
1291 & 6455 & 12911 \\
1297 & 5188 & 5189 \\
1301 & 26020 & 26021 \\
1303 & 10424 & 20849 \\
1307 & 2614 & 10457 \\
1319 & 3957 & 23743 \\
1321 & 1321 & 7927 \\
1327 & 5308 & 5309 \\
1361 & 1361 & 8167 \\
1367 & 1367 & 10937 \\
1373 & 16476 & 16477 \\
1381 & 1381 & 8287 \\
1399 & 12591 & 25183 \\
1409 & 2818 & 2819 \\
& & \\
& &
\end{tabular}

\begin{tabular}{r|r|r}
$n$ & \multicolumn{1}{|c|}{$n_{1}$} & \multicolumn{1}{|c}{$m$} \\
\hline 1423 & 5692 & 5693 \\
1427 & 8562 & 8563 \\
1429 & 5716 & 5717 \\
1433 & 4299 & 8599 \\
1439 & 1439 & 2879 \\
1447 & 17364 & 34729 \\
1451 & 1451 & 2903 \\
1453 & 5812 & 5813 \\
1459 & 1459 & 14591 \\
1471 & 5884 & 23537 \\
1481 & 2962 & 2963 \\
1483 & 7415 & 14831 \\
1487 & 8922 & 8923 \\
1489 & 14890 & 14891 \\
1493 & 10451 & 20903 \\
1499 & 1499 & 2999 \\
1511 & 1511 & 3023 \\
1523 & 3046 & 21323 \\
1531 & 3062 & 9187 \\
1543 & 6172 & 6173 \\
1549 & 6196 & 6197 \\
1553 & 4659 & 9319 \\
1559 & 1559 & 3119 \\
1567 & 6268 & 6269 \\
1571 & 3142 & 12569 \\
1579 & 6316 & 6317 \\
1583 & 1583 & 3167 \\
1597 & 6388 & 6389 \\
1601 & 3202 & 3203 \\
1607 & 9642 & 9643 \\
1609 & 3218 & 16091 \\
1613 & 4839 & 9679 \\
1619 & 6476 & 12953 \\
1621 & 3242 & 29179 \\
1627 & 4881 & 29287 \\
1637 & 31103 & 62207 \\
1657 & 6628 & 26513 \\
1663 & 6652 & 6653 \\
1667 & 1667 & 13337 \\
1669 & 3338 & 16691
\end{tabular}

\begin{tabular}{r|r|r}
\multicolumn{1}{r|}{$n$} & \multicolumn{1}{|c|}{$n_{1}$} & \multicolumn{1}{|c}{$m$} \\
\hline 1693 & 1693 & 10159 \\
1697 & 6788 & 13577 \\
1699 & 6796 & 20389 \\
1709 & 20508 & 20509 \\
1721 & 34420 & 34421 \\
1723 & 1723 & 17231 \\
1733 & 3466 & 3467 \\
1741 & 19151 & 38303 \\
1747 & 8735 & 17471 \\
1753 & 7012 & 7013 \\
1759 & 15831 & 31663 \\
1777 & 7108 & 7109 \\
1783 & 21396 & 21397 \\
1787 & 10722 & 10723 \\
1789 & 17890 & 17891 \\
1801 & 21612 & 21613 \\
1811 & 1811 & 3623 \\
1823 & 3646 & 10939 \\
1831 & 10986 & 10987 \\
1847 & 11082 & 11083 \\
1861 & 18610 & 74441 \\
1867 & 9335 & 18671 \\
1871 & 1871 & 14969 \\
1873 & 5619 & 11239 \\
1877 & 1877 & 15017 \\
1879 & 7516 & 7517 \\
1889 & 3778 & 3779 \\
1901 & 3802 & 3803 \\
1907 & 11442 & 11443 \\
1913 & 13391 & 26783 \\
1931 & 1931 & 3863 \\
1933 & 23196 & 23197 \\
1949 & 35082 & 35083 \\
1951 & 42922 & 42923 \\
1973 & 3946 & 3947 \\
1979 & 39580 & 39581 \\
1987 & 7948 & 7949 \\
1993 & 1993 & 11959 \\
1997 & 87868 & 87869 \\
1999 & 9995 & 19991 \\
& & \\
& &
\end{tabular}

\section{References}

1. ElGamal, T. (1985). A public key cryptosystem and a signature scheme based on discrete logarithms. IEEE Trans.Inform.Theory, IT-31, 469-472

2. Odlyzko, A.M. (1985). Discrete logarithms in finite fields and their cryptographic significance. Proc. of Eurocrypt'84, T.Beth, N.Cot, I.Ingemarsson (eds.), Springer LNCS 209, 224-314. 
3. Agnew, G.B., Mullin, R.C., Onyszchuk, I.M., Vanstone, S.A. (1991). An Implementation for a Fast Public Key Cryptosystem. Journal of Cryptology, 3, 63-79.

4. Bennet, C.H. (1982). The thermodynamics of computation - a review. International Journal of Theoretical Physics, 21, 12, 905-940.

5. Beth, T., Gollmann, D. (1989). Algorithm Engineering for Public Key Algorithms. IEEE JSAC, 7, 458-466.

6. Geiselmann, W., Gollmann, D. (1990). VLSI design for exponentiation in $G F\left(2^{n}\right)$. Proc. of Auscrypt'90, Seberry, J., Pieprzyk, J. (eds.), Springer LNCS 453, 398-405.

7. Haining, F.; Simple multiplication algorithms for a class of $G F\left(2^{n}\right)$. Electronics Letters, 28th March 1996; Vol 32, No 7, 636-637.

8. Hsu, I.S., Truong, T.K., Deutsch, L.J., Reed, I.S. (1988). A Comparison of VLSI Architecture of Finite Field Multipliers Using Dual, Normal, or Standard Bases. IEEE Trans. Comp., C-37, 735-739.

9. Kocher, P., Jaffe, J., Jun, B. (1999). Differential Power Analysis. Advances in Cryptology - CRYPTO'99, Wiener, M. (ed.). Springer, LNCS 1666, 388-397.

10. Koller, C.G., Athas, W.C., (1993). Adiabatic Switching, Low Energy Computing, and the Physics of Storing and Erasing Information. Proceedings of the Workshop on Physics and Computation, PhysCmp '92, IEEE Press.

11. Massey, J.L., Omura, J.K. (1981). Computational method and apparatus for finite field arithmetic. U.S. Patent application.

12. Merkle, R.C. (1993). Reversible electronic logic using switches. Nanotechnology, 4, 21-40.

13. Mullin, R.C., Onyszchuk, I.M., Vanstone, S.A., Wilson, R.M. (1988) Optimal Normal Bases in $G F\left(p^{n}\right)$. Discrete Applied Mathematics, 22, 149-161.

14. Berlekamp, E.R. (1982). Bit-Serial Reed-Solomon Encoders. IEEE Trans.Inf.Th., IT-28, 869-874.

15. Lidl, R., Niederreiter, H. (1986). Introduction to Finite Fields and Their Applications. Cambridge University Press.

16. MacWilliams, F.J., Sloane, N.J.A. (1977). The Theory of Error-Correcting Codes. North Holland. 